\title{
GRB 051028: an intrinsically faint gamma-ray burst at high redshift?^
}

\author{
1 Instituto de Astrofísica de Andalucía (IAA-CSIC), PO Box 3.004, 18080 Granada, Spain \\ e-mail: ajct@iaa.es \\ 2 Max-Planck-Institut für extraterrestrische Physik, 85748 Garching, Germany \\ 3 Max-Planck Institut für Astronomie, Koennigstuhl 17, 69117 Heidelberg, Germany \\ 4 Indian Institute of Astrophysics, 560034 Bangalore, India \\ 5 Thüringer Landessternwarte Tautenburg, Sternwarte 5, 07778 Tautenburg, Germany \\ ${ }^{6}$ Instituto de Astrofísica de Canarias, via Láctea s/n, 38205 La Laguna, Tenerife, Spain \\ 7 Laboratoire d'Astrophysique de Marseille, 13376 Marseille, France \\ 8 Nikolaev State University, Nikolskaya 24, 54030 Nikolaev, Ukraine \\ 9 American Association of Variable Star Observers, Cambridge, MA, USA
}

A. J. Castro-Tirado ${ }^{1}$, M. Jelínek ${ }^{1}$, S. B. Pandey ${ }^{1}$, S. McBreen ${ }^{2}$, J. de Jong ${ }^{3}$, D. K. Sahu ${ }^{4}$, P. Ferrero ${ }^{5}$, J. A. Caballero ${ }^{6}$, J. Gorosabel ${ }^{1}$, D. A. Kann ${ }^{5}$, S. Klose ${ }^{5}$, A. de Ugarte Postigo ${ }^{1}$, G. C. Anupama ${ }^{4}$, C. Gry ${ }^{7}$, S. Guziy ${ }^{1,8}$, S. Srividya ${ }^{4}$, L. Valdivielso ${ }^{6}$, S. Vanniarajan ${ }^{4}$, and A. A. Henden ${ }^{9}$

Received 22 December 2005 / Accepted 14 September 2006

ABSTRACT

\begin{abstract}
Aims. We present multiwavelength observations of the gamma-ray burst GRB 051028 detected by HETE-2 in order to derive its afterglow emission parameters and to determine the reason for its optical faintness when compared to other events.

Methods. Observations were taken in the optical (2.0 m Himalayan Chandra Telescope, $1.34 \mathrm{~m}$ Tautenburg, $4.2 \mathrm{~m}$ William Herschel Telescope) and in X-rays (Swift/XRT) between $2.7 \mathrm{~h}$ and $\sim 10$ days after the onset of the event.

Results. The data can be interpreted by collimated emission in a jet with a typical value of $p=2.4$ which is moving in a homogeneous interstellar medium and with a cooling frequency $v_{\mathrm{c}}$ still above the X-rays at 0.5 days after the burst onset. GRB 051028 can be classified as a "gray" or "potentially dark" GRB. On the basis of the combined optical and Swift/XRT data, we conclude that the reason for the optical dimness is not extra absorption in the host galaxy, but rather the GRB taking place at high-redshift. We also notice the very striking similarity with the optical lightcurve of GRB 050730, a burst with a spectroscopic redshift of 3.967, although GRB 051028 is $\sim 3$ mag fainter. We suggest that the bumps could be explained by multiple energy injection episodes and that the burst is intrinsically faint when compared to the average afterglows detected since 1997. The non-detection of the host galaxy down to $R=25.1$ is also consistent with the burst arising at high redshift, compatible with the published pseudo- $z$ of $3.7 \pm 1.8$.
\end{abstract}

Key words. gamma rays: bursts - techniques: photometric - cosmology: observations

\section{Introduction}

The question whether a significant fraction of gamma ray bursts (GRBs) are intrinsically faint or true dark events remains unsolved (see Filliatre et al. 2005; Castro-Tirado et al. 2006, and references therein). For instance, GRB 000418 was detected in the near-IR (Klose et al. 2000) and it is one of the reddest $(R-K=4)$ together with GRB 980329 (Reichart et al. 1999), GRB 030115 (Levan et al. 2006) and the recent GRB 050915A (Bloom \& Alatalo 2005). In most cases, it has been suggested that the cause of the reddening was dust extinction in the host galaxy. On the other hand, GRB 021211 was found to be very dim at $24 \mathrm{~h}$, as a scaled-down version of GRB 990123 (Pandey et al. 2003).

With the launch of Swift in Nov. 2004, which has the ability to follow-up the events detected by the GRB detector onboard (BAT) or by other satellites like HETE-2 and INTEGRAL, it is

* Based on observations taken with the $1.34 \mathrm{~m}$ Tautenburg telescope in Germany, with the $2.0 \mathrm{~m}$ Himalayan Chandra Telescope in India and with the $4.2 \mathrm{~m}$ William Herschel telescope at the Spanish Observatorio del Roque de los Muchachos in Canary Islands. possible to zoom in on this population of optically faint events in order to disentangle their nature.

GRB 051028 was one of such event. It was discovered by HETE- 2 on 28 Oct. 2005 , lying (90\% confidence) on a $33^{\prime} \times 18^{\prime}$ error box centred at coordinates: RA $(\mathrm{J} 2000)=01^{\mathrm{h}} 48^{\mathrm{m}} 38^{\mathrm{s}} .6$ $\operatorname{Dec}(\mathrm{J} 2000)=+47^{\circ} 48^{\prime} 30^{\prime} 0$ (Hurley et al. 2005). The burst started at $T_{0}=13: 36: 01.47 \mathrm{UT}$ and a value of $T_{90}=16 \mathrm{~s}$ is derived, putting it in the "long-duration" class of GRBs. It had a fluence of $6 \times 10^{-7} \mathrm{erg} \mathrm{cm}^{-2}$ in the $2-30 \mathrm{keV}$ range and $6 \times 10^{-6} \mathrm{erg} \mathrm{cm}^{-2}$ in the $30-400 \mathrm{keV}$ range (Hurley et al. 2005). This event was also detected by Konus/WIND in the $20 \mathrm{keV}-2 \mathrm{MeV}$ range, with a duration of $\approx 12 \mathrm{~s}$, a fluence of $\left(6.78_{-1.08}^{+0.61}\right) \times 10^{-6} \mathrm{erg} \mathrm{cm}^{-2}$ in the $20 \mathrm{keV}-2 \mathrm{MeV}$ range and a peak energy $E_{\mathrm{p}}=298_{-50}^{+73} \mathrm{keV}$ (Golenetskii et al. 2005). Swift/XRT started to observe the field $\sim 7.1 \mathrm{~h}$ after the event and detected the X-ray afterglow 5.2' away from the center of the initial HETE - 2 error box (Racusin et al. 2005).

We report here results of multi-wavelength observations in optical and X-ray waveband and discuss the reasons for the apparent optical faintness of GRB 051028 in comparison with other bursts. 
Table 1. Journal of optical observations of the GRB 051028 field.

\begin{tabular}{lcccc}
\hline \hline $\begin{array}{l}\text { Date of 2005 UT } \\
\text { (mid exposure) }\end{array}$ & $\begin{array}{c}\text { Telescope/ } \\
\text { Instrument }\end{array}$ & $\begin{array}{c}\text { Filter } \\
\text { Oct. 28, 16:18 }\end{array}$ 2.0 HCT (HFOSC) & $\begin{array}{c}\text { Exposure Time } \\
(\mathrm{s})\end{array}$ & Magnitude \\
Oct. 28, 16:32 & 2.0 HCT (HFOSC) & $R_{\mathrm{c}}$ & 300 & $20.63 \pm 0.04$ \\
Oct. 28, 16:47 & 2.0 HCT (HFOSC) & $R_{\mathrm{c}}$ & 300 & $20.72 \pm 0.05$ \\
Oct. 28, 17:03 & 2.0 HCT (HFOSC) & $R_{\mathrm{c}}$ & 300 & $21.14 \pm 0.07$ \\
Oct. 28, 17:43 & 1.34 Taut (CCD) & $R_{\mathrm{c}}$ & 1080 & $21.27 \pm 0.07$ \\
Oct. 28, 17:47 & 2.0 HCT (HFOSC) & $R_{\mathrm{c}}$ & 300 & $21.17 \pm 0.13$ \\
Oct. 28, 21:42 & 4.2 WHT (PFC) & $R$ & 300 & $21.97 \pm 0.05$ \\
Oct. 29, 05:47 & 4.2 WHT (PFC) & $R$ & 120 & $22.8 \pm 0.3$ \\
Oct. 29, 20:15 & 4.2 WHT (PFC) & $R$ & 720 & $>23.7$ \\
Oct. 31, 22:14 & 4.2 WHT (PFC) & $R$ & 2700 & $>25.1$ \\
Oct. 28, 16:25 & 2.0 HCT (HFOSC) & $I_{\mathrm{c}}$ & 300 & $19.79 \pm 0.11$ \\
Oct. 28, 16:39 & 2.0 HCT (HFOSC) & $I_{\mathrm{c}}$ & 300 & $19.94 \pm 0.06$ \\
Oct. 28, 16:55 & 2.0 HCT (HFOSC) & $I_{\mathrm{c}}$ & 300 & $20.29 \pm 0.09$ \\
Oct. 28, 17:09 & 1.34 Taut (CCD) & $I_{\mathrm{c}}$ & 1080 & $20.5 \pm 0.3$ \\
Oct. 28, 17:10 & 2.0 HCT (HFOSC) & $I_{\mathrm{c}}$ & 300 & $20.38 \pm 0.08$ \\
Oct. 28, 17:55 & 2.0 HCT (HFOSC) & $I_{\mathrm{c}}$ & 300 & $20.35 \pm 0.09$ \\
Oct. 28, 19:12 & 1.34 Taut (CCD) & $I_{\mathrm{c}}$ & 1800 & $20.67 \pm 0.23$ \\
Oct. 28, 20:33 & 1.34 Taut (CCD) & $I_{\mathrm{c}}$ & 3600 & $20.75 \pm 0.13$ \\
Oct. 28, 22:50 & 1.34 Taut (CCD) & $I_{\mathrm{c}}$ & 5400 & $21.16 \pm 0.16$ \\
Oct. 28, 18:28 & 1.34 Taut (CCD) & $V$ & 1080 & $22.08 \pm 0.20$ \\
\hline & & & &
\end{tabular}

\section{Observations and data reduction}

\subsection{X-ray observations}

We availed ourselves of the public X-ray observations from Swift/XRT which consists of four observations starting $~ 7.1$, 120,150 and $230 \mathrm{~h}$ after the event respectively. The detection in the first observation is significant (signal-to-noise ratio $S / N \sim 13.5$ ), but in later observations the X-ray afterglow is weaker and it is detected with a signal-to-noise of $3.3,2.9$ and 2.7 .

The XRT data are in photon counting mode and were reduced using the standard pipeline for XRT data using Swift software version $2.2^{1}$ and using the most recent calibration files. The data were analysed with the XSPEC version 11.3 (Arnaud 1996). Source and background regions were extracted using a circular aperture. Spectra were selected to have at least 20 counts/bin.

\subsection{Optical observations}

Target of Opportunity (ToO) observations in the optical were triggered starting $2.7 \mathrm{~h}$ after the event at the $2.0 \mathrm{~m}$ Himalayan Chandra Telescope (HCT) at Indian Astronomical Observatory (HCO). $10^{\prime} \times 10^{\prime}$ frames were taken in imaging mode with the Himalaya Faint Object Spectrograph (HFOSC), covering only the central part of the large $\left(33^{\prime} \times 18^{\prime}\right)$ HETE-2 error box. Additional observations were conducted at the $1.34 \mathrm{~m} \mathrm{Schmidt}$ telescope in Tautenburg (providing a $42^{\prime} \times 42^{\prime}$ FOV and thus covering the large error box) and at the $4.2 \mathrm{~m}$ William Herschel Telescope (WHT + Prime Focus Camera) at Observatorio del Roque de los Muchachos in La Palma (Spain). A mosaic of 2 images $\left(15^{\prime} \times 15^{\prime} \mathrm{FOV}\right)$ were taken in order to cover the entire HETE-2 error box. Subsequently, follow-up observations were taken on the following days at the $4.2 \mathrm{~m}$ WHT. Table 1 displays the observing $\log$. The optical field was calibrated using the calibration files provided by Henden (2005).

\footnotetext{
1 http://swift.gsfc.nasa.gov/docs/software/lheasoft/ download.html
}

\section{Results and discussion}

\subsection{The $X$-ray afterglow}

The X-ray data confirm the presence of a decaying X-ray source in the fraction $(70 \%)$ of the HETE-2 error box covered by the Swift/XRT, as previously reported by Racusin et al. (2005). The $\mathrm{X}$-ray position is $\mathrm{RA}(\mathrm{J} 2000)=01^{\mathrm{h}} 48^{\mathrm{m}} 15^{\mathrm{s}} \cdot 1, \operatorname{Dec}(\mathrm{J} 2000)=$ $+47^{\circ} 45^{\prime} 12^{\prime \prime} .9\left(l^{\mathrm{II}}=132.72, b^{\mathrm{II}}=-14^{\circ} .03\right)$, with an estimated uncertainty of 3.' 8 (90\% containment, Page et al. 2005).

The $\mathrm{X}$-ray light curve in the energy range 0.3 to $10 \mathrm{keV}$ is shown in Fig. 1. The early X-ray light curve $\left(2 \times 10^{4}\right.$ to $\left.5 \times 10^{4} \mathrm{~s}\right)$ can be fit by a power-law decay $F_{\mathrm{X}} \propto t^{\alpha_{\mathrm{X}}}$ with exponent $\alpha_{\mathrm{X}}=-1.43 \pm 0.60$ with a $\chi^{2} /$ d.o.f. $=9.3 / 10$. The data were also fit including the late time data up to 10 days $\left(\sim 8.6 \times 10^{5} \mathrm{~s}\right)$ and resulted in a exponent $\alpha_{\mathrm{X}}=-1.1_{-0.2}^{+0.15}\left(\right.$ with $\chi^{2} /$ d.o.f. $\left.=10.7 / 13\right)$ compatible with the power-law index obtained considering only the early observations. The value of $\alpha_{\mathrm{X}}$ is dominated by the late time data and a break or flattening of the light curve at intervening times is possible and cannot be excluded by the observations.

A spectrum was extracted for the first observation starting at $7.1 \mathrm{~h}$ consisting of 5 Swift orbits. The X-ray spectrum was fit by an absorbed power-law with photon index $\Gamma=2.3_{-0.25}^{+0.30}$ with a column density $N_{\mathrm{H}}=0.40_{-0.25}^{+0.30} \times 10^{22} \mathrm{~cm}^{-2}$ (with $\chi^{2} /$ d.o.f. $\left.=9.1 / 9\right)$ (Fig. 2). The galactic column density, $N_{\mathrm{H}, \mathrm{GAL}}$, was estimated to be $1.2 \times 10^{21} \mathrm{~cm}^{-2}$ using the weighted average of 6 points within $1^{\circ}$ of the source location ${ }^{2}$ (Dickey \& Lockman 1990). The values used to estimate $N_{\mathrm{H}, \mathrm{GAL}}$ range from $1.01 \times 10^{21} \mathrm{~cm}^{-2}$ to $1.33 \times 10^{21} \mathrm{~cm}^{-2}$. The fitted spectrum is compatible at $90 \%$ confidence level with Galactic absorption of $1.2 \times 10^{21} \mathrm{~cm}^{-2}$ (Fig. 3). A power-law index of $\Gamma=1.7 \pm 0.2\left(\chi^{2} /\right.$ d.o.f. $\left.=12.8 / 10\right)$ (i.e. a spectral $\mathrm{X}$-ray index $\beta_{\mathrm{X}}=-0.7 \pm 0.2$ with $\left.F(v) \propto v^{\beta}\right)$ is obtained if only Galactic absorption $N_{\mathrm{H}, \mathrm{GAL}}$ of $1.2 \times 10^{21} \mathrm{~cm}^{-2}$ is considered in agreement with Page et al. (2005). Alternatively, if we assume that all of the extra absorption originates in the host galaxy and freeze the $N_{\mathrm{H}, \mathrm{GAL}}$ at $1.2 \times 10^{21} \mathrm{~cm}^{-2}$ then the intrinsic absorption

\footnotetext{
${ }^{2}$ http://heasarc.gsfc.nasa.gov/cgi-bin/Tools/ w3nh/w3nh.pl
} 


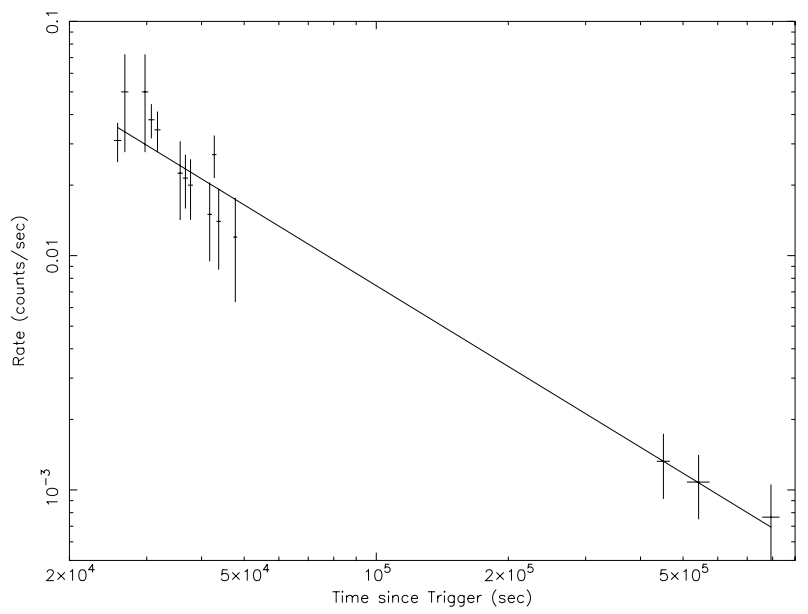

Fig. 1. The X-ray lightcurve obtained by Swift/XRT starting $7.1 \mathrm{~h}$ after the event onset and continuing up to 10 days later. The data are fit by a power-law decline exponent $\alpha_{\mathrm{X}}=-1.1_{-0.2}^{+0.1}$.

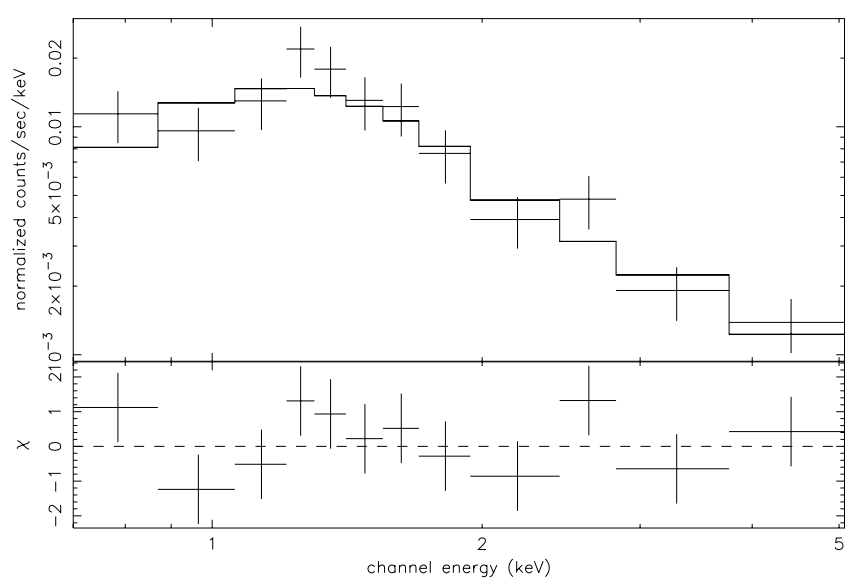

Fig. 2. The X-ray spectrum obtained by Swift/XRT for the time interval $T_{0}+7.1 \mathrm{~h}$ to $T_{0}+13.2 \mathrm{~h}$. The data can be fitted by a power-law with photon index $\Gamma=2.3_{-0.25}^{+0.30}$.

in the host at the pseudo- $z$ (see below) of $z=3.7$ is $N_{\mathrm{H}, z=3.7}$ of $12.2_{-12.1}^{+18.5} \times 10^{22} \mathrm{~cm}^{-2}$.

\subsection{The optical afterglow}

The optical counterpart was discovered on our $R$-band images taken at the $4.2 \mathrm{~m}$ WHT telescope starting $7.5 \mathrm{~h}$ after the onset of the gamma-ray event. A faint $R=21.9$ object was detected inside the Swift/XRT error circle (Jelínek et al. 2005; Pandey et al. 2005). Astrometry against USNO-B yielded the coordinates: $\mathrm{RA}(\mathrm{J} 2000)=01^{\mathrm{h}} 48^{\mathrm{m}} 15^{\mathrm{s}} \cdot 00, \operatorname{Dec}(\mathrm{J} 2000)=+47^{\circ} 45^{\prime} 09^{\prime} \cdot 4$, with 0.2 uncertainty ( $1 \sigma$, see Fig. 4$)$.

With $E(B-V)=0.21$ in the line of sight (Schlegel et al. 1998), $A_{V}=0.71$ is derived (which translates into $A_{V}=0.6$ if the correction factor proposed by Dutra et al. (2003) is taken into account). A value of $A_{V}=0.7$ is obtained using the fit from Predehl \& Schmitt (1995) for the Galactic H column. We choose $A_{V} \sim 0.7$ for the rest of this paper, which implies $A_{R}=0.53$ and $A_{I}=0.37$.

From the analysis of the full VRI dataset available obtained at Hanle, Tautenburg and La Palma, we have obtained the optical afterglow lightcurve plotted in Fig. 5. The data between $T_{0}+$ $4 \mathrm{~h}$ and $T_{0}+15 \mathrm{~h}$ can be fitted by a shallow power-law decline

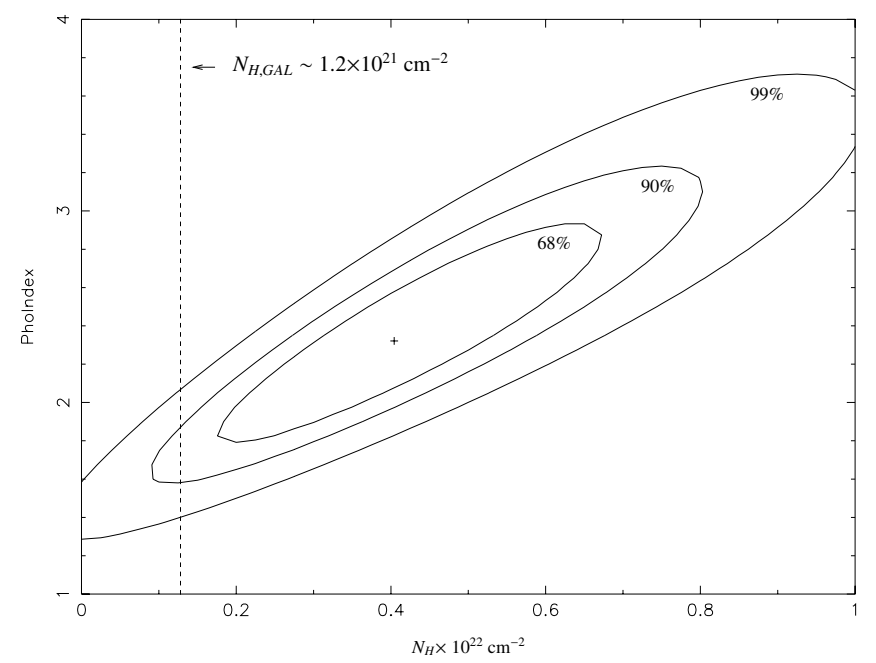

Fig. 3. Contour plot of column density versus photon index for the absorbed power law model shown in Fig. 2. The dashed line shows the estimated Galactic column density value $1.2 \times 10^{21} \mathrm{~cm}^{-2}$ and the contours denote $68 \%, 90 \%$ and $99 \%$ confidence levels respectively. The spectrum is compatible ( $90 \%$ confidence) with Galactic absorption.

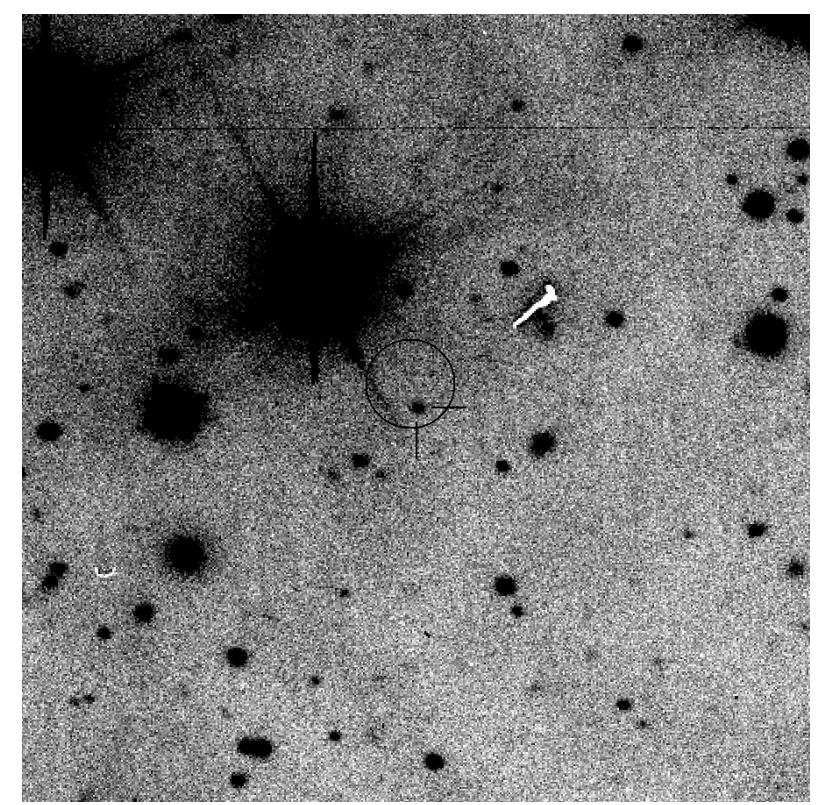

Fig. 4. The deep $R$ band image of the GRB 051028 field taken at the 4.2WHT on 28 Oct. 2005. The optical afterglow within the 3'. 8 Swift/XRT error box (circle) is depicted. The field is $2.5 \times 1{ }^{\prime} .9$ with North up and East to the left.

with decay index $\alpha_{\text {opt }}=-0.9 \pm 0.1$. The upper limits obtained at 1.5 and 3.5 day ( $>23.7$ and $>25.1$ respectively) may suggest the existence of a break in the lightcurve after $\sim 1$ day.

The data prior to $4 \mathrm{~h}$ (i.e. in the range $T_{0}+2.7 \mathrm{~h}$ and $T_{0}+4 \mathrm{~h}$ ) show a bumpy behaviour very similar to the one seen in other events like GRB 021004 (de Ugarte Postigo et al. 2005), GRB 030329 (Guziy et al. 2006, and references therein) and GRB 050730 (Pandey et al. 2006). In fact, the similarity with GRB 050730 is very remarkable, if GRB 051028 is shifted up by 3 mag (Fig. 6). There is evidence for at least two of such bumps taking place, superimposed on the power-law decline. This could be explained in the framework of multiple energy injection episodes (Björnsson et al. 2004). GRB 050730 is an 


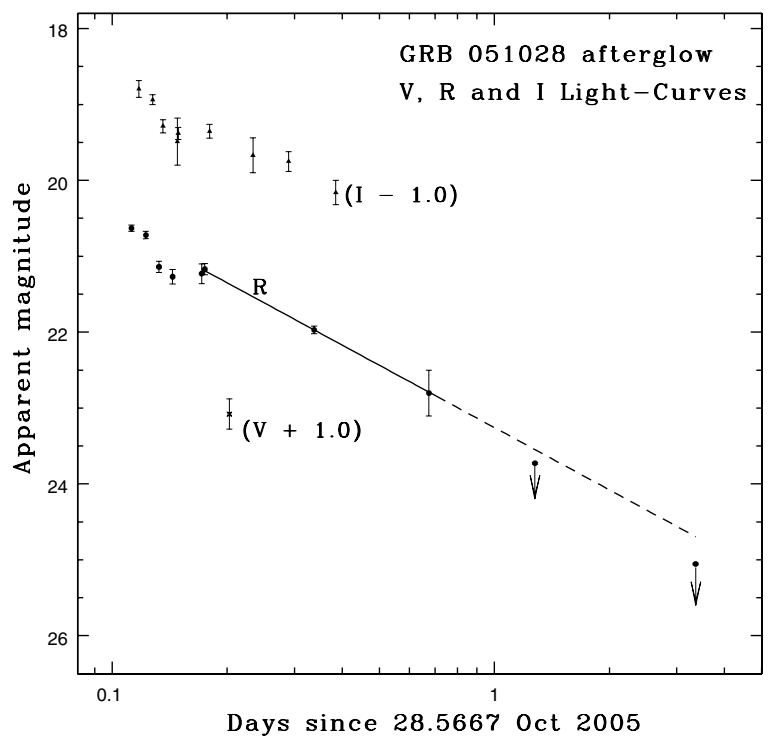

Fig. 5. The $R$ and $I$-band lightcurves (including the $V$-band single detection) obtained at Hanle (HCT), Tautenburg and La Palma (WHT) starting $2.7 \mathrm{~h}$ after the event onset and continuing up to 3.5 days later. The data after $4.0 \mathrm{~h}$ are fit by a power-law decline exponent $\alpha_{\mathrm{opt}}=-0.9 \pm 0.1$.

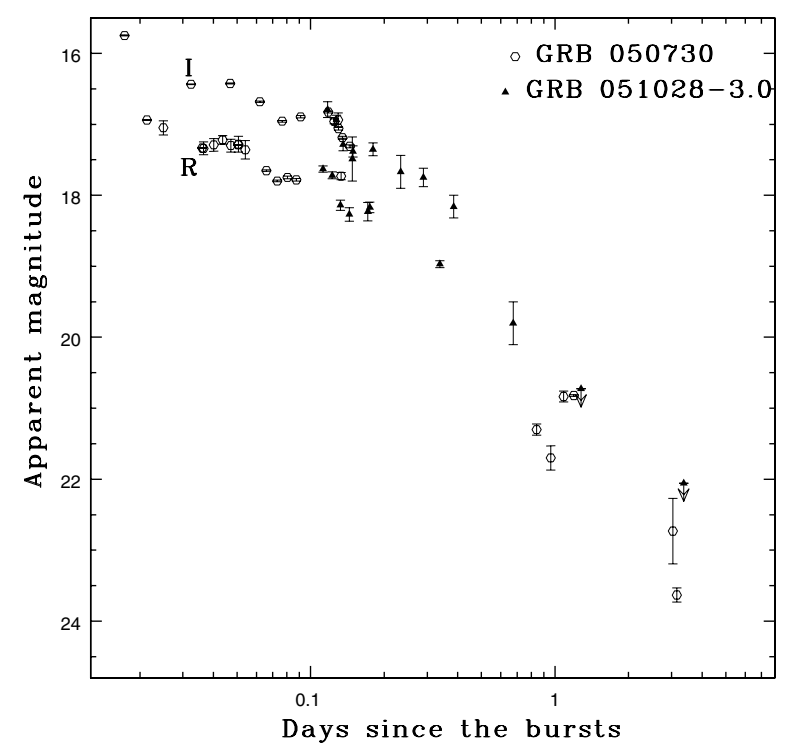

Fig. 6. The GRB $051028 R$ and $I$-band light curves shifted by $\sim 3$ mag in order to match the GRB $050730 R$-band lightcurve (from Pandey et al. 2006). No shift in the $T-T_{0}$ values has been performed. These combined data strengthen the evidence of a "bumpy" behaviour of the GRB 051028 afterglow.

optically bright afterglow (see Fig. 12 of Nardini et al. 2005) whereas GRB 051028 seems an optically faint event if at redshift $z \sim 3-4$. Unfortunately there is no X-ray data available at this epoch to allow a more complete modelling being carried out.

\subsection{A high redshift event}

We have extrapolated the optical and X-ray fluxes of the GRB 051028 afterglow to $T_{0}+11 \mathrm{~h}$ and derived a value of $\beta_{\text {opt }-\mathrm{X}}=-0.55 \pm 0.05$. Thus GRB 051028 is located in the

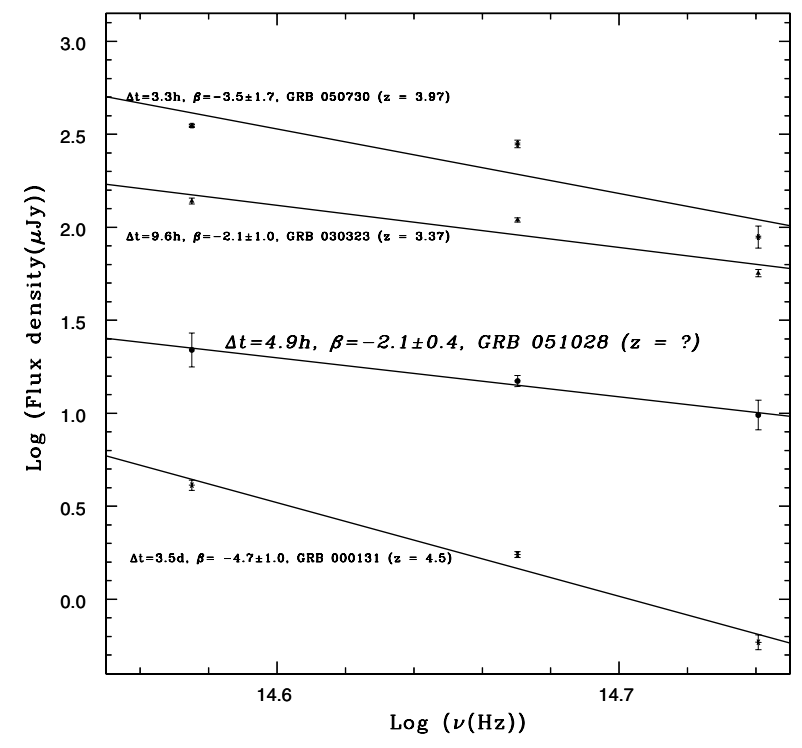

Fig. 7. The $\beta_{\text {opt }}$ spectral indexes obtained for GRB optical afterglows in the redshift range $3<z<4.5$ based solely on the VRI data. The $\beta_{\text {opt }}$ value derived for GRB 051028 is in the range of those derived for this high- $z$ sample and therefore supports that GRB 051028 also arose at a $z=3-4$.

"gray" or "potentially dark" GRB locus on the dark GRB diagram by Jakobsson et al. (2004). How can the optical faintness of GRB 051028 be explained?

Although the redshift of this event could not be properly measured due to its faintness at the time of the discovery, we are able to constrain it on the basis of the VRI-band data presented in this paper. Using the magnitudes derived here and correcting them for the Galactic extinction in the line of sight, we determine a spectral optical index $\beta_{\mathrm{opt}}=-2.1 \pm 0.4$. In the simplest fireball models (Sari et al. 1998), $F_{v} \propto v^{\beta}$ with $\beta=-p / 2$ for $v>v_{\mathrm{c}}$ and $\beta=-(p-1) / 2$ for $v<v_{\mathrm{c}}$. Thus, for a typical range of $p$ values in the range $1.5<p<3$ (Zeh et al. 2006), $\beta_{\text {opt }}$ should be in the range $-1.5<\beta_{\text {opt }}<-0.25$. In fact, the GRB 051028 X-ray data before $T_{0}+0.5$ day are well fitted by a jet model with $p=2.4$ in the slow cooling case, moving through the ISM (with $\rho=$ constant) prior to the jet break time and with a cooling frequency $v_{\mathrm{c}}$ still above the X-rays. A value of $\Gamma=1.7$ is favoured (as $\Gamma=2.3$ is giving high, unrealistic values of $p$ ) and thus we can consider that all the absorption is Galactic in origin (and ruling out dust along the line of sight in the host galaxy). The X-ray data (both values of $\Gamma$ ) are also eventually fitted for a value of $p=2.1$ if $v_{\mathrm{c}}$ would have already crossed the X-ray band at that time $(0.5 \mathrm{~d})$, as it seems to be derived from a sample of events studied by BeppoSAX (Piro et al. 2005), but this is unlikely in the light of the recent Swift/XRT results for a sample of (presumably higher- $z$ ) events (Panaitescu et al. 2006). In any of the above mentioned cases, the observed value of $\alpha_{\text {opt }}$ can be reproduced and therefore $\beta_{\mathrm{opt}}$ should be $\sim-0.7$. What is the reason for the discrepancy in the observed and expected values of $\beta_{\text {opt }}$ ?

Figure 7 shows the derived $\beta_{\text {opt }}$ when using only VRI magnitudes for a sample of bursts in the range $3.3<z<4.5$. As can be seen the derived values are in the range of the one found for GRB 051028, well above the $\beta_{\mathrm{opt}}=1.5$ value mentioned previously. This is naturally explained by the fact that at $z \sim 3.2$ and $\sim 4.0$, the Lyman- $\alpha$ break begins affecting the $V$ and $R$ passbands respectively. Therefore, one natural explanation for 
the $\beta_{\text {opt }}$ value found for GRB 051028 is that it also arose at a $z \approx 3-4$, a value to be compared with that of GRB $050730(z=$ 3.967), a burst which has a suprisingly similar optical afterglow lightcurve, as we have shown in Sect. 3.2. This $z \approx 3-4$ value is in fact in agreement with the pseudo- $z=3.7 \pm 1.8$ derived for this burst using the recent pseudo- $z$ estimator developed by Pélangeon et al. (2006) on the basis of the observed peak energy and the bolometric luminosity in the $15 \mathrm{~s}$ long interval containing the highest fluence. This would be in agreement with the fact that no host galaxy is detected down to $R=25.1$. This high redshift is also supported by the late break time, as typical afterglows undergo a jet break episode before $T_{0}+1$ day in the rest frame (Zeh et al. 2006). In fact, the Ghirlanda et al. (2004) $E_{p}-E_{\gamma}$ relation is satisfied for GRB 051028 when considering the pseudo- $z=3.7$.

The fact that the afterglow of GRB 051028 is not unusual in the Swift/XRT sample may indicate that the density of the surrounding medium where the progenitor has taken place should be closer to the the typical value of $\approx 1 \mathrm{~cm}^{-3}$ derived for several long-duration GRBs. So a low density environment is not the reason for its faintness at optical wavelengths. A possibility is that GRB 051028 could be an underluminous GRB similar to GRB 980613, GRB 011121 and GRB 021211 (see Nardini et al. and references therein), in contrast to GRB 050730.

\section{Conclusions}

We have presented multiwavelength observations of the long duration GRB 051028 detected by HETE-2 between $2.7 \mathrm{~h}$ and $\sim 10$ days after the event. The X-ray afterglow of GRB 051028 can be compared to other GRB afterglows in the sense that its flux at $11 \mathrm{~h}$ is typical, i.e., one can assume that the burst has occurred on a classical $n \sim 1 \mathrm{~cm}^{-3}$ environment. The optical afterglow, on the other hand, is dim at a similar epoch (and comparable for instance to GRB 030227 , Castro-Tirado et al. 2003). We also noticed the remarkable similarity to the optical afterglow of GRB 050730, a burst lasting $\sim 10$ times longer with comparable gamma-ray fluence ${ }^{3}$ at $z=$ 3.967 (see Pandey et al. 2006, and references therein). This indicates that the faintness of the optical emission is not due to a lowdensity environment as in the case of some short GRBs, such as GRB 050509b (Castro-Tirado et al. 2005). Instead, we propose that GRB 051028 occurred in a faint galaxy (with $R>25.1$ ) at a high redshift consistent with the pseudo- $z=3.7 \pm 1.8$.

Thanks to the extraordinary repointing capabilities of Swift, the accurate localisations for future events and the corresponding multiwavelength follow-up will shed more light on the origin of this faint optical afterglow population.

Acknowledgements. We thank the anonymous referee for useful suggestions. This research has made use of data obtained through the High Energy Astrophysics Science Archive Research Center On line Service, provided by the NASA/Goddard Space Flight Center. Publically available Swift/XRT data are also acknowledged. P.F., D.A.K. and S.K. thanks financial support by DFG grant K1 766/13-2. This research has also been partially supported by the Ministerio de Ciencia y Tecnología under the programmes AYA2004-01515 and ESP200204124-C03-01 (including FEDER funds). SMB acknowledges the support of the European Union through a Marie Curie Intra-European Fellowship within the Sixth Framework Program.

\section{References}

Arnaud, K. A. 1996, ASP Conf. Ser., 101, 17

Bloom, J. S., \& Alatalo, K. 2005, GCN Circ., 3984

Björnsson, G., Gudmundsson, E. H., \& Jóhannesson, G. 2004, ApJ, 715, L77

Castro-Tirado, A. J., Gorosabel, J., Guziy, S., et al. 2003, A\&A, 411, 315

Castro-Tirado, A. J., de Ugarte Postigo, A., Gorosabel, J., et al. 2005, A\&A, 439, L15

Castro-Tirado, A. J., Bremer, M., McBreen, S., et al. 2006, A\&A, submitted

Dickey, J. M., \& Lockman, F. J. 1990, ARA\&A, 28, 215

Dutra, C. M., Ahumada, A. V., Clariá, J. J., Bica, E., \& Barbuy, B. 2003, A\&A, 408, 287

Filliatre, P., D’Avanzo, P., Covino, S., et al. 2005, A\&A, 438, 793

Ghirlanda, G., Ghisellini, G., \& Lazzati, D. 2004, A\&A, 616, 331

Golenetskii, S., Aptekar, R., Mazets, E., et al. 2005, GCN Circ., 4183

Guziy, S., et al. 2006, in preparation

Henden, A. A. 2005, GCN Circ., 4184

Jakobsson, P., Hjorth, J., Fynbo, J. P. U., et al. 2004, ApJ, 617, L21

Klose, S., Stecklum, B., Masetti, N., et al. 2000, ApJ, 545, 271

Hurley, K., Ricker, G., Atteia, J.-L., et al. 2005, GCN Circ., 4172

Jelinek, M., Pandey, S. B., Guziy, S. S., et al. 2005, GCN Circ., 4175

Levan, A., Fruchter, A., Rhoads, J., et al. 2006, ApJ, in press

Nardini, M., Ghisellini, G., Ghirlanda, G., et al. 2006, A\&A, 451, 821

Page, K. L., Goad, M. R., Burrows, D. N., et al. 2005, GCN Circ., 4205

Panaitescu, A., Mészáros, P., Burrows, D., et al. 2006, MNRAS, 369, 2059

Pandey, S. B., Anupama, C. G., Sagar, R., et al. 2003, A\&A, 408, L21

Pandey, S. B., Jelinek, M., Guziy, S. S., et al. 2005, GCN Circ., 4176

Pandey, S. B., Castro-Tirado, A. J., McBreen, S., et al. 2006, A\&A, in press [arXiv:astro-ph/0607471]

Pélangeon, A., Atteia, J.-L., Lamb, D. Q., et al. 2006, in Gamma-ray bursts in the Swift Era, ed. S. S. Holt, N. Gehrels, \& J. A. Nousek (New York), AIP Conf. Proc., 836, 149

Piro, L. 2005, Nuovo Cimento 28C, 473

Predhel, P., \& Schmitt, J. H. M. M. 1995, A\&A, 293, 889

Racusin, J., Page, K., Kennea, J., et al. 2005, GCN Circ., 4174

Reichart, D. E., Lamb, D. Q., Metzger, M. E., et al. 1999, ApJ, 517, 692

Sari, R., Piran, T., \& Narayam, R. 1998, ApJ, 497, L17

Schlegel, D. J., Finkbeiner, D. P., \& Davis, M. 1998, ApJ, 500, 525

de Ugarte Postigo, A., Castro-Tirado, A. J., Gorosabel, J., et al. 2005, A\&A, 443, 841

Zeh, A., Klose, S., \& Kann, D. A. 2006, ApJ, 637, 889
3 The scarcity of the available X-ray data for GRB 051028 does not allow to make a straigth comparison with respect to the GRB 050730 X-ray afterglow. 